\title{
TOROIDAL ARCS ARE CELLULAR ${ }^{1}$
}

\section{TOM KNOBLAUCH}

ABSTRACT. We prove that a toroidal, cell-like, locally connected continuum is cellular.

1. Introduction. An arc may be a decreasing intersection of cubeswith-two-handles and still not be cellular, or even toroidal. An arc formed by joining two Fox-Artin arcs [4] at their tame ends serves as an example.

However it follows directly from the theorem below that all toroidal arcs are cellular. The theorem generalizes Daverman's result [3] concerning toroidal 3-cells. It was suggested to me as a problem by D. R. McMillan.

2. Definitions. A continuum $X$ in a 3-manifold $M^{3}$ is cellular if $X=\bigcap_{i=1}^{\infty} X_{i}$ where $X_{i+1} \subseteq$ Int $X_{i}$ for each $i$, and each $X_{i}$ is a 3-cell in $M^{3}$.

A continuum $X$ in $M^{3}$ is toroidal if $X=\bigcap_{i=1}^{\infty} X_{i}$ where $X_{i+1} \subseteq$ Int $X_{i}$ for each $i$, and each $X_{i}$ is a solid torus in $M^{3}$.

A continuum $X$ in $M^{3}$ is cell-like if for any neighborhood $U$ of $X$ in $M^{3}$, there is a neighborhood $V$ of $X$ in $M^{3}$ such that $V$ is homotopically trivial in $U$.

3. THEOREM. A toroidal, cell-like, locally connected continuum is cellular.

Proof. Assume the continuum $X$ is toroidal and cell-like but not cellular. Then $X=\bigcap_{i=0}^{\infty} T_{i}$ where for each $i, T_{i}$ is a solid torus and $T_{i+1} \subseteq$ Int $T_{i}$. Since $X$ is cell-like, we may assume that for each $i$ the winding number of $T_{i+1}$ in $T_{i}$ is zero (that is, $T_{i+1}$ is homotopically trivial in $T_{i}$ ). Since $X$ is not cellular, we may assume that for each $i$ the wrapping number of $T_{i+1}$ in $T_{i}$ is not zero (that is, each meridional disk of $T_{i}$ intersects $\left.T_{i+1}\right)$. Let $D, E, F$, and $G$ be four disjoint polyhedral meridional disks of $T_{0}$ with $F$ and $G$ in different components of $T_{0}-(D \cup E)$. We may assume that for each $i, T_{i}$ is polyhedral and $\mathrm{Bd} T_{i}$ is in general position with $\Delta=D \cup E \cup F \cup G . \Delta \cap \mathrm{Bd} T_{i}$ is a finite collection of trivial and meridional (with respect to $\mathrm{Bd} T_{i}$ ) simple closed curves, because the wrapping number of $T_{i}$ in $T_{0}$ is not zero [2, Theorem 1].

Received by the editors August 13, 1971.

AMS 1970 subject classifications. Primary 57A60.

Key words and phrases. Cellularity, toroidal continuum, cell-like continuum.

1 This research was supported by an NSF Fellowship.

c. American Mathematical Society 1972 
If $\Delta \cap \mathrm{Bd} T_{1}$ has any trivial simple closed curves on $\mathrm{Bd} T_{1}$, choose $J$ to be an innermost trivial curve on $\mathrm{Bd} T_{1} . J$ bounds a disk $D^{\prime}$ on $\mathrm{Bd} T_{1}$ whose interior misses $\Delta$.

$J$ lies in one of the four disks $D, E, F$, or $G$, say $D$, and bounds a disk $D^{\prime \prime}$ there. Replace $D$ by $\left(D-D^{\prime \prime}\right) \cup D^{\prime}$ and then push $D^{\prime}$ slightly off Bd $T_{1}$ to the appropriate side. We can remove all trivial curves in this way. We have four new disjoint meridional disks $D_{1}, E_{1}, F_{1}$, and $G_{1}$.

Similarly change $\left(D_{1}, E_{1}, F_{1}, G_{1}\right)$ to $\left(D_{2}, E_{2}, F_{2}, G_{2}\right)$ so that if $\Delta_{2}=$ $D_{2} \cup E_{2} \cup F_{2} \cup G_{2}$ then $\Delta_{2} \cap \mathrm{Bd} T_{j}$ contains no trivial simple closed curves of $\mathrm{Bd} T_{j}$ for $j \leqq 2$.

Continue this process to get a sequence $\left(D_{1}, E_{1}, F_{1}, G_{1}\right), \cdots$, $\left(D_{n}, E_{n}, F_{n}, G_{n}\right), \cdots$ where for each $n$, if $\Delta_{n}=D_{n} \cup E_{n} \cup F_{n} \cup G_{n}$ then (Bd $\left.T_{j}\right) \cap \Delta_{n}$ contains no trivial curves of $\operatorname{Bd} T_{j}$ for $J \leqq n$.

We use this construction to prove the following lemma.

LEMMA. There are infinitely many components of $X-\Delta$ each of whose closures intersect two of the disks $D, E, F$, and $G$.

Proof. It is clearly enough to show that given $n>0$ there are at least $n$ such components. It is also enough to show that given $n>0, \exists m>0$ such that there are at least $n$ components of $X-\Delta_{m}$ whose closures intersect two of $D_{m}, E_{m}, F_{m}$, and $G_{m}$. In fact, each component of $X-\Delta_{m}$ whose closure intersects, say, $D_{m}$ and $F_{m}$ contains a component of $X-\Delta$ whose closure intersects $D$ and $F$. To see this let $C$ be a component of $X-\Delta_{m}$ such that $\bar{C}$ intersects $D_{m}$ and $F_{m}$. Then $\bar{C}$ intersects $D$ and $F$ since $\Delta_{m} \cap X \subseteq \Delta \cap X$. By a theorem of elementary topology $\bar{C}$ contains an irreducible continuum $C^{\prime}$ from $D$ to $F$ and $C^{\prime}-(D \cup F)$ is connected. The component of $X-\Delta$ containing $C^{\prime}-(D \cup F)$ lies in $C$ and its closure intersects $D$ and $F$.

Now, fixing $N>0$, consider a homotopy core $J$ of $T_{N}$ lying in $\operatorname{Bd} T_{N}$. Also take $J$ so that it intersects each curve of $\left(B d T_{N}\right) \cap \Delta_{N}$ just once. $J$ must contain a subarc from $D_{N}$ to $E_{N}$. Without loss of generality assume this arc lies in the $F_{N}$ half of $T_{0}-\left(D_{N} \cup E_{N}\right)$. The existence of this arc assures the existence of a cylinder in $\mathrm{Bd} T_{N}$ with one end in $D_{N}$ and one in $E_{N}$. A spanning cylinder is an annulus with interior in the $F_{N}$ half of $T_{0}-\left(D_{N} \cup E_{N}\right)$ and with one boundary component in $D_{N}$ and one in $E_{N}$. Spanning cylinders are defined only for the integer $N$. A spanning cylinder $A$ is said to be inside a spanning cylinder $B$ if $D_{N} \cup E_{N} \cup B$ separates Int $A$ from $\mathrm{Bd} T_{0}$. Inside $B$ is the bounded closed component of $T_{0}$ $\left(D_{N} \cup E_{N} \cup B\right)$. Choose an outermost spanning cylinder $C_{N, 1} \subseteq \operatorname{Bd} T_{N}$. $C_{N, 1}$ must lie inside an outermost spanning cylinder $C_{N-1,1} \subseteq \mathrm{Bd} T_{N-1}$. The following linking argument assures the existence of another outermost spanning cylinder $C_{N, 2} \subseteq \mathrm{Bd} T_{N}$ inside $C_{N-1,1}$. 
Suppose $C_{N .1}$ is the unique outermost spanning cylinder of $\mathrm{Bd} T_{N}$ inside $C_{N-1,1}$. Let $J_{1}=C_{N, 1} \cap D_{N}$ and $J_{2}=C_{N-1,1} \cap D_{N}$. Pull $J$ slightly off $C_{N, 1}$ to the inside of $T_{N}$. Then we still have $J \subseteq T_{N} . J_{1}$ is a meridian of Bd $T_{N}$, so the disk in $D_{N}$ bounded by $J_{1}$ contains an odd number of points of $J . J_{2}$ is a meridian of $\mathrm{Bd} T_{N-1}$, so since the winding number of $T_{N}$ in $T_{N-1}$ is zero, the disk in $D_{N}$ bounded by $J_{2}$ contains an even number of points of $J$. Then the annulus in $D_{N}$ bounded by $J_{1}$ and $J_{2}$ contains an odd number of points of $J$. However, by the uniqueness assumption, each point of $J$ in the annulus is the endpoint of subarc of $J$ which runs from $D_{N}$ into the $F_{N}$ half of $T_{0}-\left(D_{N} \cup E_{N}\right)$ and back to $D_{N}$ again. Therefore the number of points of $J$ in the annulus is even, a contradiction.

Now $C_{N-1,1}$ is inside an outermost spanning cylinder $C_{N-2,1}$ of $T_{N-2}$. The linking argument gives us another outermost spanning cylinder $C_{N-1,2}$ of Bd $T_{N-1}$ inside $C_{N-2,1}$. After $N$ applications of the linking argument we have spanning cylinders $C_{i, j}, 1 \leqq i \leqq N$ and $j \leqq 2$, where each $C_{i, j} \subseteq \operatorname{Bd} T_{i}$ and is an outermost such cylinder. In addition $C_{i, j}$ is inside $C_{i-1,1}$ for $1<i \leqq N$. Thus

$$
\text { inside } C_{i, 2} \cap \text { inside } C_{j, 2}=\varnothing \quad \text { for } i \neq j \text {. }
$$

The lemma will be proved if we can find a component of $X-\Delta_{N}$ inside each $C_{i, 2}$ whose closure intersects $F_{N}$ and one of $D_{N}$ or $E_{N}$. If we knew $X \cap F_{N} \cap$ inside $C_{i, 2} \neq \varnothing$, then we could find an irreducible continuum $C^{\prime}$ in $X$ from $F_{N}$ ninside $C_{i, 2}$ to $\left(D_{N} \cup E_{N}\right)$ ninside $C_{i, 2}$. The component of $X-\Delta_{N}$ containing $C^{\prime}-\Delta_{N}$ would be the desired component. Then we need only show $X \cap F_{N}$ ninside $C_{i, 2} \neq \varnothing$. Take an innermost (in $F_{N}$ ) simple closed curve $J$ of $F_{N} \cap C_{i, 2} . J$ is meridional on $T_{i}$ and bounds a disk $H$ in $F_{N}$ which intersects $X$ only if $F_{N}$ does inside $C_{i, 2}$. Choose an innermost (in $H$ ) curve $J^{\prime}$ of $H \cap B d T_{i}$. $J^{\prime}$ bounds a disk $H^{\prime}$ in $H$ and inside $C_{i, 2} . H^{\prime}$ is a meridional disk of $T_{i}$ so $H^{\prime} \cap X \neq \varnothing$ and therefore $X \cap F_{N} \cap$ inside $C_{i, 2} \neq \varnothing$ and the lemma is proved.

The theorem now follows easily from the lemma. There are infinitely many components of $X-\Delta$ whose closures intersect two of these disks, say $D$ and $F$. It follows easily that $X$ is not locally connected.

4. Corollary. Suppose $X$ is a cell-like, locally connected continuum in $S^{3}$ and $X=\bigcap_{i=0}^{\infty} X_{i}$ where $X_{i+1} \subseteq$ Int $X_{i}$ and each $X_{i}$ is a 3-manifold bounded by a torus ( $a$ solid torus or a cube-with-a-knotted-hole). Then $X$ is cellular.

Proof. Assume $X$ satisfies the hypotheses of the corollary but $X$ is not cellular. By the theorem $X$ is not toroidal, so we may assume each $X_{i}$ is a cube-with-a-knotted-hole [1]. Since $X$ is cell-like we may assume $X_{i+1}$ is homotopically trivial in $X_{i}$ for each $i$. Since $X$ is not cellular we 
may assume no $X_{i}$ contains a cell with $X$ in its interior. Also take each $X_{i}$ to be polyhedral.

Now let $T_{i}=S^{3}-\operatorname{Int} X_{i}$ for each $i$. Then $T_{i} \subseteq \operatorname{Int} T_{i+1}$ and each $T_{i}$ is a knotted solid torus (this is the definition of a cube-with-a-knotted-hole).

Property 1. For each $i$, there is no meridional disk of $T_{i+1} \operatorname{missing} T_{i}$. For if there were such a polyhedral disk $D$, a closed regular neighborhood $N\left(X_{i+1} \cup D\right)$ would be a cell in $X_{i}$ containing $X$ in its interior.

Property 2. $T_{i}$ is homotopically trivial in $T_{i+1}$, or $T_{i} \sim 0$ in $T_{i+1}$. Since $X_{i+1} \sim 0$ in $X_{i}$, if $J$ is a meridional simple closed curve of $T_{i+1}$, then $J \sim 0$ in $S^{3}-T_{i}$. So the winding number of $T_{i}$ in $T_{i+1}$ is zero, so $T_{i} \sim 0$ in $T_{i+1}$.

However Kister and McMillan [5] showed that the union of an ascending sequence of knotted solid tori with the Properties 1 and 2 cannot be imbedded in $S^{3}$. The corollary is thus proved by contradiction.

\section{REFERENCES}

1. J. W. Alexander, On the subdivision of 3-space by a polyhedron, Proc. Nat. Acad. Sci. U.S.A. 10 (1924), 6-8.

2. R. H. Bing, Point-like decompositions of $E^{3}$, Fund. Math. 50 (1961/62), 431-453. MR 25 \#560.

3. R. J. Daverman, On the number of nonpiercing points in certain crumpled cubes, Pacific J. Math. 34 (1970), 33-43. MR 42 \#6801.

4. R. H. Fox and E. Artin, Some wild cells and spheres in three-dimensional space, Ann. of Math. (2) 49 (1948), 979-990. MR 10, 317.

5. J. M. Kister and D. R. McMillan, Jr., Locally Euclidean factors of $E^{4}$ which cannot be imbedded in $E^{3}$, Ann. of Math. (2) 76 (1962), 541-546. MR 26 \#1868.

Department of Mathematics, University of Wisconsin, Madison, Wisconsin 53706 